\title{
Electron-interface phonon interaction in multiple quantum well structures
}

\author{
J P Sun $\dagger$, H B Teng $\dagger$, G I Haddad $\dagger$ and M A Stroscio $\ddagger$ \\ † Center for High Frequency Microelectronics, Solid State Electronics Laboratory, \\ Department of Electrical Engineering and Computer Science, \\ The University of Michigan, Ann Arbor, MI 48109-2122, USA \\ ‡ US Army Research Office, PO Box 12211, Research Triangle Park, \\ NC 27709-2211, USA
}

Received 7 December 1997, accepted for publication 11 March 1998

\begin{abstract}
Intersubband relaxation rates due to electron interactions with the interface phonons are evaluated for multiple quantum well structures designed for step quantum well lasers operating at mid-infrared to submillimetre wavelengths. The interface phonon modes and electron-phonon interaction Hamiltonians for the structures are derived using the transfer matrix method, based on the macroscopic dielectric continuum model, whereas the electron wavefunctions are obtained by solving the Schrödinger equation. Fermi's golden rule is employed to calculate the electron relaxation rates between the subbands in these structures. The relaxation rates for two different structures are examined and compared with those calculated using the bulk phonon modes and the Fröhlich interaction Hamiltonian. The sum rule for the relationship between the form factors of the various localized phonon modes and the bulk phonon modes is verified. The results obtained in this work illustrate that the transfer matrix method provides a convenient way for deriving the properties of the interface phonon modes in different structures of current interest and that, for preferential electron relaxation in intersubband laser structures, the effects of the interface phonon modes are significant and should be considered for optimal design of these laser structures.
\end{abstract}

\section{Introduction}

In recent years, several quantum well structures have been proposed and demonstrated for achieving lasing wavelengths from the mid-infrared to the submillimetre wave region [1-4]. These lasers incorporate narrow quantum wells that must have thicknesses as small as 30-50 $\AA$ and have transition level separations engineered to one unit or a multiple of the longitudinal-optical (LO) phonon energy. The level separations are critical design parameters for these laser structures, because preferential relaxation rates between these levels are required to maintain adequate carrier population inversion between the active laser states and successful lasing operation at the designed wavelength. It is well known that electron-phonon interactions are the major physical processes for electron relaxation from the upper energy levels to the lower levels. However, similar to the modification of electron behaviour in confined structures, it is now well recognized that the shapes and energies of phonon modes are modified by quantum wells. Specifically, LO phonons in quantum wells may be described by the dielectric continuum model in terms of confined and interface (IF) modes that have properties different from those of bulk phonons [5-8]. There is clearly a need to formulate the phonon modes localized in these quantum well structures and to determine how these localized phonon modes affect electron transitions or relaxation and how they influence laser performance.

Two laser structures shown in figures 1 and 2 are studied: a symmetric quantum well structure and an asymmetric quantum well structure, consisting of $\mathrm{Al}_{0.6} \mathrm{Ga}_{0.4} \mathrm{As}$ as the outermost layers, $\mathrm{Al}_{0.25} \mathrm{Ga}_{0.75} \mathrm{As}$ as the step layers and the GaAs quantum well. These two structures represent basic structural cells used in novel laser structures such as the step quantum well lasers [4]. The quantities of interest in this study include the relevant confined, IF and bulk-like modes, the bound-state electronic energies and wavefunctions and the significant phonon-assisted transition rates, or the relaxation rates, for these structures. These relaxation rates are also important in quantum cascade lasers [1] and tunnelling injection lasers [2].

\section{Solution method}

We outline in this section the solution procedures of the relaxation rate calculations based on the theory of localized phonons in quantum well systems. The main formulations employed and developed in this work are also presented.

\subsection{Dielectric continuum model}

Mori and Ando [8], in their study of electron-optical phonon interaction in heterostructures, derived the interaction Hamiltonian using orthonormal eigenmodes of phonons calculated in the dielectric continuum model. 


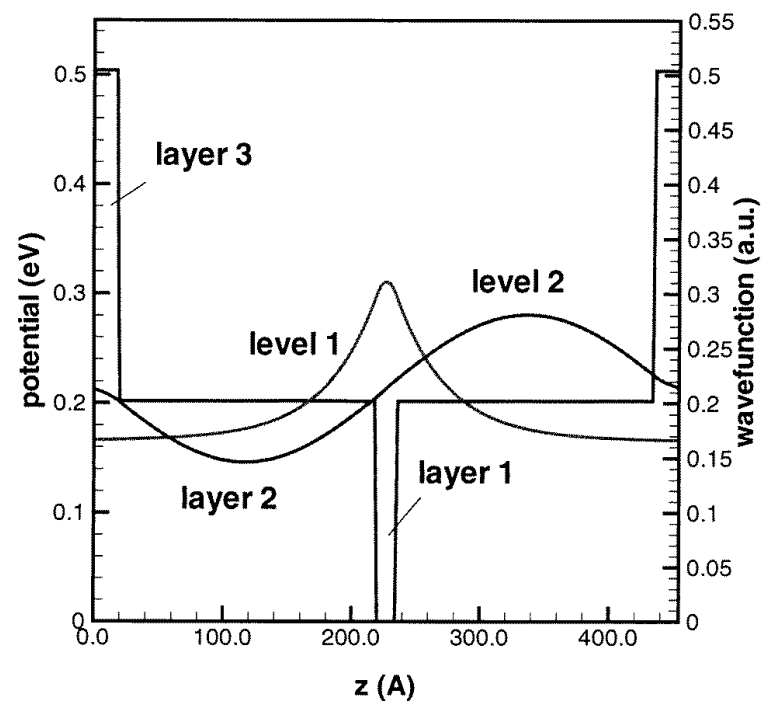

Figure 1. Symmetric quantum well structure: electron potential and calculated wavefunctions of the first two levels.

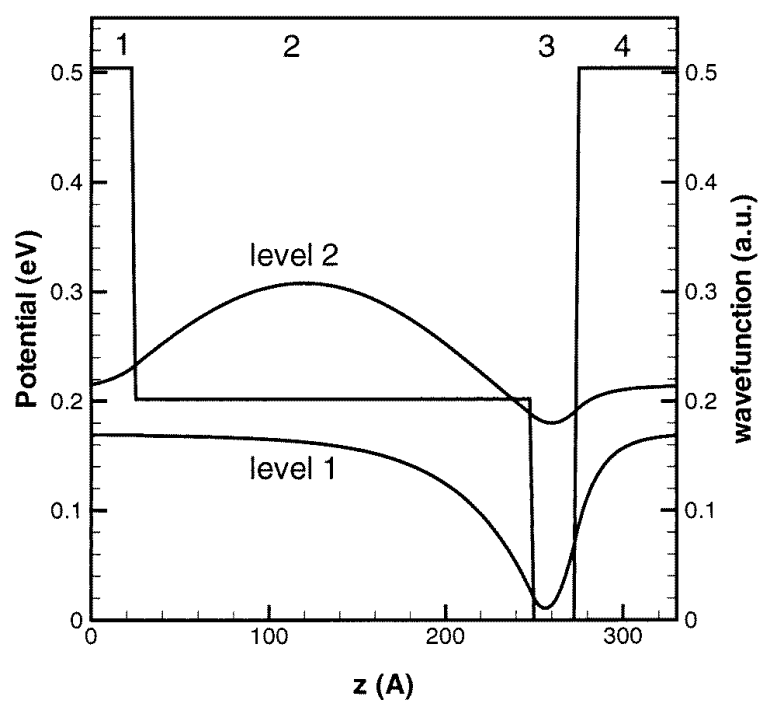

Figure 2. Asymmetric quantum well structure: electron potential and calculated wavefunctions of the first two levels. The numbers 1, 2, 3, 4 denote the different regions.

The dielectric continuum model determines optical phonon modes using classical electrostatics by solving the following equations in each layer of the heterostructure

$$
\begin{gathered}
\nabla^{2} \Phi(\boldsymbol{r})=\frac{1}{\epsilon_{0}} \nabla \cdot \boldsymbol{P}(\boldsymbol{r}) \\
\boldsymbol{P}(\boldsymbol{r})=\epsilon_{0} \chi_{n}(\omega) \boldsymbol{E}(\boldsymbol{r}) \\
\boldsymbol{E}(\boldsymbol{r})=-\nabla \Phi(\boldsymbol{r})
\end{gathered}
$$

with the boundary conditions at the heterointerfaces. In equations (1)-(3), $\Phi(\boldsymbol{r})$ is the electrostatic potential as a function of the three-dimensional position vector $r$ due to the optical phonon modes, $\boldsymbol{P}(\boldsymbol{r})$ the polarization field, $\boldsymbol{E}(\boldsymbol{r})$ the electric field, $\epsilon_{0}$ the permittivity of vacuum and $\chi_{n}(\omega)=\epsilon_{n}(\omega)-1$ the dielectric susceptibility in layer $n$.
The dielectric function $\epsilon_{n}(\omega)$ is given by [9]

$$
\epsilon_{n}(\omega)=\epsilon_{n}(\infty) \frac{\omega^{2}-\omega_{L}^{2}}{\omega^{2}-\omega_{T}^{2}}
$$

for a binary compound semiconductor, or

$$
\epsilon_{n}(\omega)=\epsilon_{n}(\infty) \frac{\left(\omega^{2}-\omega_{L A}^{2}\right)\left(\omega^{2}-\omega_{L B}^{2}\right)}{\left(\omega^{2}-\omega_{T A}^{2}\right)\left(\omega^{2}-\omega_{T B}^{2}\right)}
$$

for a ternary compound semiconductor, following the generalized Lyddane-Sachs-Teller relation, where the subscript $L$ denotes the LO phonon modes and $T$ the transverse-optical (TO) phonon modes, respectively, and $\epsilon_{n}(\infty)$ is the high-frequency dielectric constant.

For a given heterojunction system the normal LO phonon modes (mode frequencies and dispersion relationships) and the corresponding $\Phi(\boldsymbol{r}), \boldsymbol{P}(\boldsymbol{r})$ and $\boldsymbol{E}(\boldsymbol{r})$ can be obtained by solving equations (1)-(3) simultaneously with the boundary conditions at the heterointerfaces. More details of the solution procedures can be found in [8]. In their study of single heterostructures, three types of normal phonon modes have been identified: (1) IF modes, (2) half-space LO modes and (3) half-space TO modes. For double heterostructures, there are six types of modes: (1) symmetric IF modes, (2) antisymmetric IF modes, (3) confined LO modes, (4) confined TO modes, (5) halfspace LO modes and (6) half-space TO modes. We will investigate properties of these modes and their influence on the electron relaxation process in the laser structures.

\subsection{Relaxation rates and interaction Hamiltonian}

For evaluation of electron relaxation rates between the subbands, Fermi's golden rule is employed. The relaxation rate or transition probability per unit time by the electron-LO phonon interaction is given by

$$
W_{i f}=\frac{2 \pi}{\hbar}\left|\left\langle f\left|\mathcal{H}_{i n t}\right| i\right\rangle\right|^{2} \delta\left(\boldsymbol{E}_{f}-\boldsymbol{E}_{i} \pm \hbar \omega\right)
$$

where $\mathcal{H}_{\text {int }}=-e \Phi$ is the interaction Hamiltonian and $e$ the magnitude of electronic charge. $i$ and $f$ denote the electron initial and final state, respectively. $\omega$ is the phonon frequency. Once the Hamiltonians and wavefunctions are known, the relaxation rates can be calculated by numerical integration of equation (6). For the bulk cases, $\mathcal{H}_{\text {int }}$ is in the well-known Fröhlich form, whereas, in the heterostructures, changes of the Fröhlich Hamiltonian due to phonon confinement and localization must be evaluated. For simple heterostructures, the interaction Hamiltonians can be readily derived in analytical form from the localized phonon modes based on the dielectric continuum model. However, for more complicated structures such as those involving multiple quantum wells or mixed binary-ternary material systems, dozens of localized phonon modes exist and the interaction Hamiltonians become very complicated and have to be treated numerically, as performed in this work.

\subsection{Form factors and sum rule}

The interaction Hamiltonians are derived from the normal phonon modes and are therefore dependent on the 
device structure. However, the terms in the interaction Hamiltonian which are independent of material parameters such as the phonon frequencies and dielectric constants can be grouped and defined to be the form factors [8]. Because of the complete orthonormality of the eigenmodes, a sum rule exists. For example, for the double heterostructures,

$$
F_{B}(q)=F_{S}(q)+F_{A}(q)+F_{C}(q)+F_{H^{+}}(q)+F_{H^{-}}(q)
$$

where $F_{B}(q)$ is the form factor in the case where the electrons are assumed to interact with bulk LO phonons and $q$ is the in-plane wavevector, $F_{A}$ is for the asymmetric IF phonon modes, $F_{S}$ for the symmetric IF modes, $F_{C}$ for the confined modes and $F_{H^{+}}$and $F_{H^{-}}$are for the halfspace phonons in the right and left half-space, respectively. A major advantage of the sum rule is that one can justify the calculations of the various phonon modes using equation (7). We made use of the sum rule in this work to verify our calculations.

\subsection{Transfer matrix method}

The various localized phonon modes, the related interaction Hamiltonians and the relaxation rates for simple heterostructures such as single or double quantum wells composed of binary semiconductors may be derived in analytical forms for further computation. This has been done by Stroscio [10] for a symmetric quantum well structure. However, for heterosystems consisting of multiple heterointerfaces or asymmetric quantum wells, application of even a simple macroscopic dielectric continuum model becomes highly complicated owing to the coupling of adjacent interfaces. In addition, ternary or quaternary composition will add further complexity to the phonon modes. Yu et al [11] developed a general transfer matrix formalism to determine the electrostatic potential and dispersion relations of the IF phonons in a multiple-IF heterostructure within the framework of the dielectric continuum model. In this work, the transfer matrix method is employed to generate the IF phonon modes and numerical Hamiltonians for the asymmetric structure. The analysis follows the procedures described in [11]. For a given IF phonon mode, the electrostatic potential $\Phi(\boldsymbol{r})$ in the region $\left[z_{i}, z_{i+1}\right]$ and its two-dimensional Fourier transform $\Phi_{i}(\boldsymbol{q}, z)$ are defined by

$$
\begin{gathered}
\Phi_{i}(\boldsymbol{r})=\sum_{q} \exp (-\mathrm{i} \boldsymbol{q} \cdot \boldsymbol{\rho}) \Phi_{i}(\boldsymbol{q}, z) \\
\Phi_{i}(\boldsymbol{q}, z)=c_{i-} \mathrm{e}^{-q z}+c_{i+} \mathrm{e}^{+q z}
\end{gathered}
$$

where $\boldsymbol{\rho}=(x, y)$ and $\boldsymbol{q}$ denote the positions and wavevectors in the two-dimensional plane of the interface. The $z$-axis is chosen as the direction of growth. Specifically, for the asymmetric structure,

$$
\Phi= \begin{cases}\Phi_{1} & -\infty<z<0 \\ \Phi_{2} & 0 \leq z<a \\ \Phi_{3} & a \leq z<b \\ \Phi_{4} & b \leq z<+\infty\end{cases}
$$

where for the regions $i=1,2,3,4$ (see figures 1 and 2), $\Phi_{i}$ is given by

$$
\Phi_{i}=A\left(C_{i-} \mathrm{e}^{-q z}+C_{i+} \mathrm{e}^{q z}\right)
$$

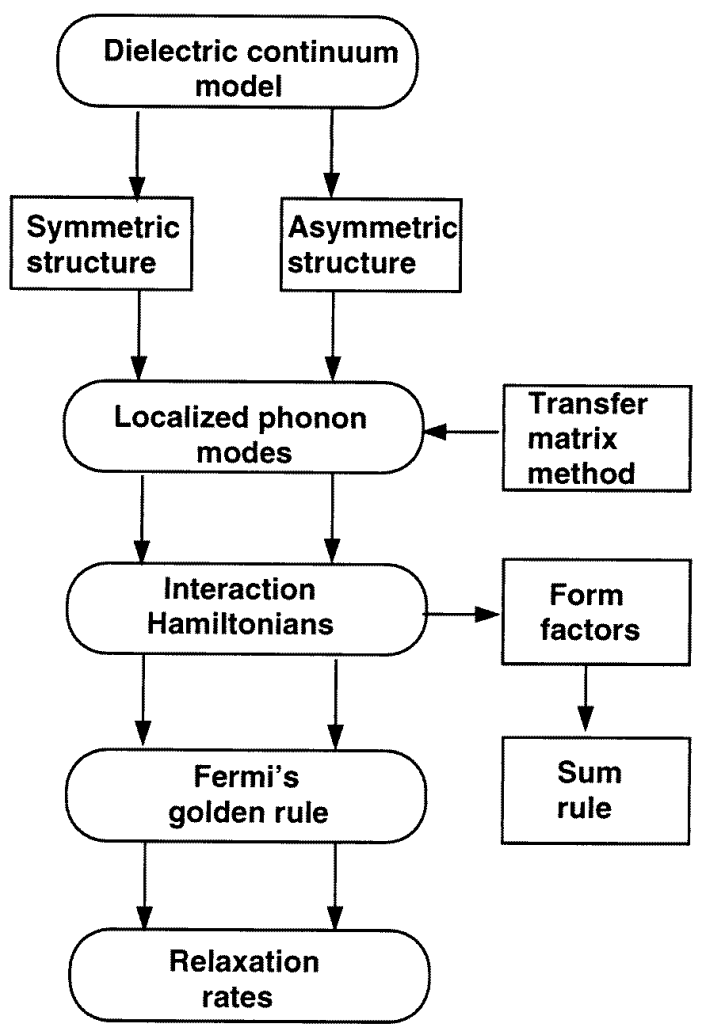

Figure 3. Outline of solution procedures employed and developed in this work.

where the normalization factor $A$ is derived to be

$$
A=\left[\left.\sum_{i} \frac{\epsilon_{0}}{\hbar} \frac{\partial \epsilon_{i}(\omega)}{\partial \omega} q\left(C_{i+}^{2} \mathrm{e}^{2 q z}-C_{i-}^{2} \mathrm{e}^{-2 q z}\right)\right|_{z_{i}} ^{z_{i+1}}\right]^{-1 / 2}
$$

and the coefficients $C_{i \pm}$ are functions of structural parameters and phonon in-plane wavevectors and are obtained by the transfer matrices set by the boundary conditions at the interfaces. The dispersion relations of the phonon modes are given by the solutions of

$$
\begin{aligned}
\left(\epsilon_{4}-\right. & \left.\epsilon_{3}\right)\left(\epsilon_{3}+\epsilon_{2}\right)\left(\epsilon_{2}-\epsilon_{1}\right) \mathrm{e}^{-2 q b} \\
& +\left(\epsilon_{4}-\epsilon_{3}\right)\left(\epsilon_{3}-\epsilon_{2}\right)\left(\epsilon_{2}+\epsilon_{1}\right) \mathrm{e}^{-2 q(b-a)} \\
& +\left(\epsilon_{4}+\epsilon_{3}\right)\left(\epsilon_{3}-\epsilon_{2}\right)\left(\epsilon_{2}-\epsilon_{1}\right) \mathrm{e}^{-2 q a} \\
& +\left(\epsilon_{4}+\epsilon_{3}\right)\left(\epsilon_{3}+\epsilon_{2}\right)\left(\epsilon_{2}+\epsilon_{1}\right)=0 .
\end{aligned}
$$

The solution procedures employed and developed in this work are outlined in figure 3.

\section{Results and summary}

For the symmetric laser structure, evaluation of the various localized phonon modes and their effects on the relaxation rates has been carried out based on the formulations of the phonon modes and interaction Hamiltonians developed by Stroscio [10]. However, the analytical approximations for electron wavefunctions at the different subbands used in [10] are replaced with more accurate wavefunctions obtained by numerically solving the Schrödinger equation for this structure. The relaxation rates between the 


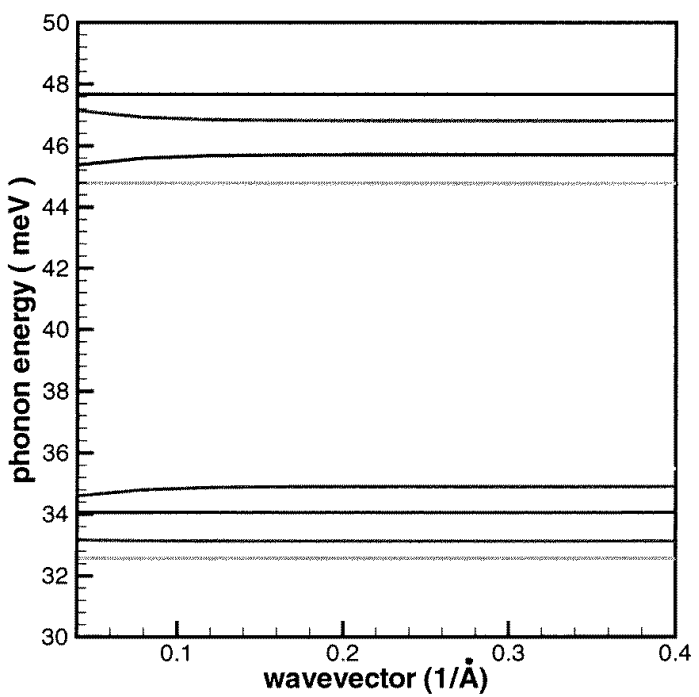

Figure 4. Calculated IF phonon modes in the asymmetric structure based on the transfer matrix method. Note that two of the modes are merged together.

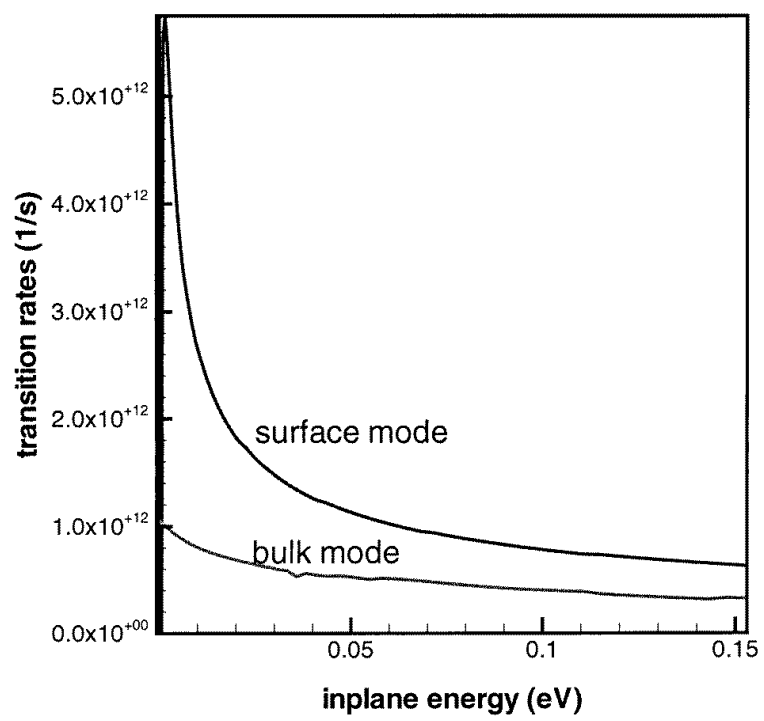

Figure 5. Transition rates as functions of electron in-plane energy in the asymmetric structure.

subbands are calculated using Fermi's golden rule with numerical integration. The electron relaxation rates are found to be dominated by the IF phonon scattering. In particular, the antisymmetric IF-phonon-assisted transition dominates at a level separation close to the IF phonon frequency $\left(E_{2}-E_{1}=47 \mathrm{meV}\right)$. A maximum or resonant relaxation rate was found when the electron in-plane energy compensates the difference between the mode frequency and energy level separation. A device structure designed to have an energy level separation of the antisymmetric IF mode frequency would have a relaxation rate several times larger than that designed with the bulk phonon frequency. In order to justify our calculations, the sum rule of the form factors is verified numerically for various energy levels. The sum of the form factors of the various phonon modes added together is equal to that of the bulk mode. The

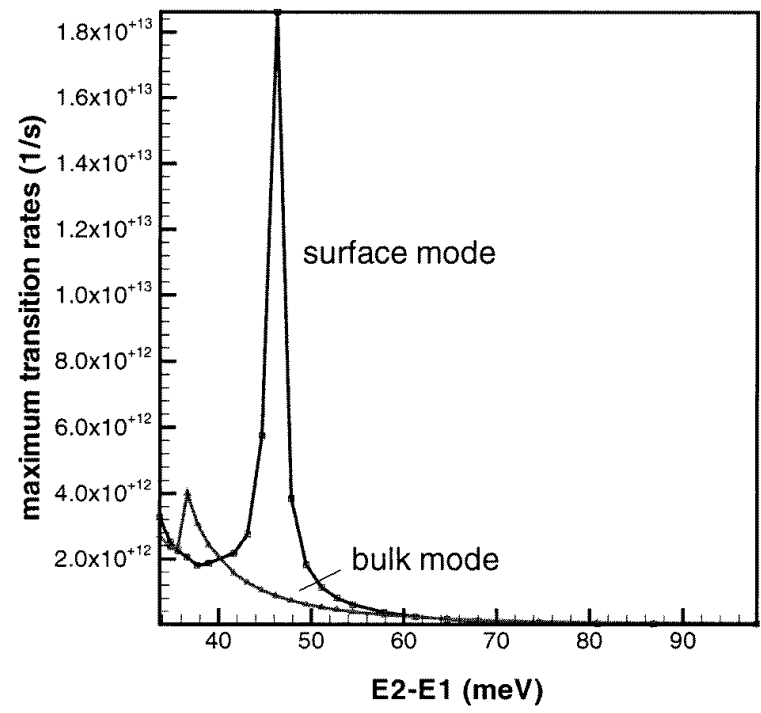

Figure 6. Resonant transition rates as functions of energy level separation in the asymmetric structure.

agreement is excellent. More details of the findings on the symmetric structure are described elsewhere [12].

Effects of the IF phonon on electron relaxation for the asymmetric structure have also been calculated. There are 10 branches of the IF phonon modes in their dispersion relations, as shown in figure 4. Unlike the symmetric structure, the asymmetric structure does not produce distinctive symmetric and antisymmetric IF phonon branches in the dispersion spectrum. We have therefore referred to these phonon modes as the surface modes. In figure 5, the IF-phonon-assisted relaxation rates are plotted versus the electron in-plane energy. It is seen that the relaxation rate assisted by the surface phonons is approximately 6 times as large as that of the bulk phonon. Finally, in figure 6 , the resonant relaxation is shown to have a sharp increase at the IF phonon energy and to have a higher relaxation rate as compared with the case of the symmetric structure.

In summary, evaluations of localized phonon effects on the electron relaxation rates in the novel quantum well laser structures performed in this work have shown that, to facilitate preferential electron relaxation for optimal laser performance, it is important to include design considerations of electron transitions induced by the IF phonons and to have electron subbands engineered with their separation close to the interface phonon energy. Further work in this direction may lead to more optimized design modifications for novel quantum well laser devices.

\section{Acknowledgment}

This work was supported by the US Army Research Office Contract DAAL03-91-C-0034.

\section{References}

[1] Faist J, Capasso F, Sivco D L, Sirtori C, Hutchinson A L and Cho A Y 1994 Quantum cascade laser Science 264 553 
[2] Sun H C, Davis L, Sethi S, Singh J and Bhattacharya P 1993 Properties of a tunneling injection quantum-well laser: recipe for a 'cold' device with a large modulation bandwidth IEEE Photon. Tech. Lett. 5870

[3] Afzali-Kushaa A, Haddad G I and Norris T B 1993 Optically pumped intersubband lasers Int. Semiconductor Device Research Symp. (Charlottesville, VA, 1-3 December 1993)

[4] Zhang X, Haddad G I, Sun J P, Afzali-Kushaa A and Sung C Y 1995 Population inversion in step quantum wells at $10 \mu \mathrm{m}$ wavelength 53rd IEEE Device Research Conf. (Charlottesville, VA, 19-21 June 1995)

[5] Fuchs R and Kliewer K L 1965 Optical modes of vibration in an ionic crystal slab Phys. Rev. A 1402076

[6] Lucas A A, Kartheuser E and Badro R G 1970 Electron-phonon interaction in dielectric films: application to electron energy loss and gain spectra Phys. Rev. B 22488

[7] Licari J J and Evrard R 1977 Electron-phonon interaction in a dielectric slab: effect of the electronic polarizability Phys. Rev. B 152254

[8] Mori N and Ando T 1989 Electron-optical-phonon interaction in single and double heterostructures Phys. Rev. B 406175

[9] Kim K W and Stroscio M A 1990 Electron-optical-phonon interaction in binary/ternary heterostructures $J$. Appl. Phys. 686289

[10] Stroscio M A 1996 Interface-phonon-assisted transitions in quantum-well lasers J. Appl. Phys. 806864

[11] Yu S, Kim K W, Stroscio M A, Iafrate G J, Sun J P and Haddad G I 1997 Transfer matrix method for interface optical phonon modes in multiple-interface heterostructure system J. Appl. Phys. 823363

[12] Sun J P, Teng H B, Haddad G I, Stroscio M A and Iafrate $\mathrm{G} \mathbf{J}$ Intersubband relaxation in step quantum well structures International Workshop on Computational Electronics (Notre Dame, IN, May 1997), VSLI Des. to be published 